\title{
Binary Star Stellar Population Synthesis Model For Astrophysical Studies
}

\author{
Z. M. Li, C. Y. Mao, L. Chen and Q. Zhang \\ Institute for Astronomy and History of Science and Technology, Dali University, China \\ email: zhongmu.li@gmail.com
}

\begin{abstract}
Binary stars possibly exists in most galaxies and star clusters. Their evolution can lead to significant change in stellar population studies. Binary star to fit group (BS2fit: $\infty$ ) has built up a binary star stellar population synthesis model and used it in a few works. This page is to introduce the model and its possible applications.
\end{abstract}

Keywords. binary stars, stellar populations, galaxies, star clusters

\section{Introduction}

Stellar population is an important tool for astrophysical studies, and populations are usually modeled by single stars. However, binaries have significant effects on stellar population synthesis. Therefore, it is necessary to build and use population models that have taken the effects of binaries into account. BS2fit group gives an example.

\section{Stellar population model for both binary and single stars: RPS}

\subsection{Model inputs, outputs and features}

Some reliable and widely used results are taken for the initial mass function, mass range, stellar evolution and spectral library of our model, i.e., rapid stellar population synthesis model (RPS). The outputs include isochrones, color-magnitude diagrams, colors, spectra, and line indices.

There are four main feature for RPS model (Li \& Han 2008, Li et al. 2012, Li et al. 2013): 1) It reproduces special stars such as blue stragglers, yellow stragglers and red stragglers. 2) It can reproduce special CMD features of star clusters, broad main sequences, extended turn-offs, special stars, dual or extended red clumps. 3) It shows UV-upturn spectra for old simple stellar populations (SSPs). 4) It changes the results of both SSP and composite stellar population (CSP) studies. In particular, our results show that population models with binary evolution will report significantly different star formation histories compared to previous works.

\section{Potential applications}

The model can be used for studies such as spectral fitting, line index fitting, CMD studies, color studies of galaxies and star clusters, as a special and powerful tool.

\section{References}

Li, Z. M. \& Han, Z. W. 2008, MNRAS, 387, 105

Li, Z. M., Mao C. Y., Chen, L., \& Zhang, Q., 2012, ApJL, 761, L22

Li, Z. M., Mao C. Y., Chen, L., Zhang, Q. Li, \& M. C. 2013, arXiv, 1302.0927 